\title{
Síndrome depresivo en la adolescencia asociado a género, abuso sexual, violencia física y psicológica
}

\author{
Cristina Haydée Arrom Suhurt ${ }^{\mathrm{I}}$, Margarita Samudio ${ }^{\mathrm{I}}$, Monica Ruoti ${ }^{\mathrm{I}}$, Elizabeth Orú $^{\mathrm{I}}$ \\ I. Instituto de Investigaciones en Ciencias de la Salud-Universidad Nacional de Asunción (IICS-UNA), \\ Paraguay
}

Cómo referenciar este artículo/ How to reference this article:
Arrom C, Samudio M, Ruoti M, Orúe E. Síndrome depresivo en la adolescencia asociado a género, abuso sexual, violencia física y violencia. Mem. Inst. Investig. Cienc. Salud. 2015;13(3):39-44.

\section{RE S U M E N}

El objetivo fue identificar factores asociados al síndrome depresivo en adolescentes de colegios públicos y privados del departamento de Alto Paraná. Se realizó un estudio transversal en adolescentes de 56 colegios (públicos y privados) de las 4 ciudades más pobladas del departamento (Hernandarias, Presidente Franco, Minga guazú, Ciudad del Este). Se completó un cuestionario auto-administrado, estructurado y anónimo, previa autorización de los padres. Para conocer la sintomatología referida al síndrome depresivo se utilizaron 20 preguntas referidas a niveles de malestar psíquicos utilizadas en la encuesta nacional "Salud Mental y Hábitos tóxicos en Paraguay". La presencia de 11 síntomas como minino era indicador de la presencia de depresión. Las variables estudiadas fueron sexo, edad, antecedente de violencia psicológica, antecedente de violencia física, intento de abuso sexual o abuso consumado. El 35,8\% $(n=237)$ de los 661 estudiantes presentaba sintomatología depresiva. En el análisis bivariado, los factores asociados a la depresión fueron sexo femenino (valor $p<0,0001$; OR: 2,99), antecedente de violencia física ( $p<0,0001 ;$ OR: 2,48$)$, antecedente de violencia psicológica $(p<0,0001 ;$ OR: 4,04), intento de abuso sexual $o$ abuso consumado $(p<0,0001 ;$ OR: 2,3$)$. En el análisis multivariado los factores asociados fueron antecedente de violencia psicológica $(p<0,001$; OR: 4,3$)$, el sexo femenino $(p<0,001$; OR: 3,5$)$, intento de abuso sexual o abuso consumado ( $p<0,001 ;$ OR: 2,0$)$. Los factores asociados a la depresión después del ajuste estadístico fueron violencia psicológica, sexo femenino y abuso sexual.

Palabras clave: depresión, adolescencia, factores asociados.

\section{Depressive syndrome in adolescence associated with gender, sexual abuse, physical and psychological violence}

\begin{abstract}
A B S T R A C T
The objective was to identify factors associated with depressive syndrome in adolescents from public and private schools of the department of Alto Parana. A cross-sectional study was conducted in 56 adolescents of the 4 largest cities of the department (Hernandarias, Presidente Franco, Minga Guazú, Ciudad del Este). A self-administered, structured and anonymous questionnaire was completed after parental consent. To know the symptomatology related to depressive syndrome 20 questions related to psychological distress levels used in the national survey "Mental Health and Toxic habits in Paraguay" were used. The presence of 11 symptoms as minimum was indicative of the presence of depression. The variables studied were sex, age, history of psychological violence, attempt of sexual abuse or committed sexual abuse. The 35.8\% $(n=237)$ of the 661 students had depressive symptoms. In bivariate analysis, factors associated with depression were female ( $p<0.0001$; OR: 2.99), a history of physical violence ( $p$ value $<0.0001$; OR: 2.48), history of psychological violence ( $p$ value $<0.0001, \mathrm{OR}=4.04$ ), attempted sexual assault or committed abuse $(p<0.0001 ; O R=2.3)$. In the multivariate analysis, associated factors
\end{abstract}


were history of psychological violence $(p<0.001 ;$ OR: 4.3$)$, female sex $(p<0.001 ;$ OR: $3.5)$, attempt of sexual abuse or committed abuse $(p<0.001$; OR: 2.0$)$. The factors associated with depression after statistical adjustment was psychological violence, female gender and sexual abuse.

Keywords: depression, adolescence, associated factors

\section{INTRODUCCIÓN}

Según datos de la Organización Mundial de la Salud (OMS Pardo) la prevalencia global de trastornos mentales a nivel mundial afecta a aproximadamente 851.000 .000 personas e incluye los trastornos neuróticos, afectivos, retardo mental, epilepsia, demencias y esquizofrenia. Cifras del Banco Mundial señalan que para el año 2020, la depresión mayor será la segunda causa de carga de enfermedad en el mundo, (1).

La depresión es un problema que no es detectado la mayoría de las veces en la adolescencia (2) y sus altos índices, según señalan Gómez y Rodríguez, pueden verse afectados por condiciones socioeconómicas, altos índices de desempleo, violencia, pobreza, incertidumbre laboral y pocas expectativas ocupacionales. En la infancia y la adolescencia hay notas diferenciales con respecto a este trastorno del estado de ánimo en la edad adulta. Más allá de que aumente la incidencia de los trastornos depresivos entre la población adolescente, su prevalencia es suficientemente digna de consideración y oscila entre un 15 y un $13 \%$, citado por Pardo A. Graciela (1).

Los jóvenes constituyen uno de los grupos etarios que presentan mayor probabilidad de sufrir depresión, ya que en la etapa de la adolescencia se llevan a cabo procesos de cambio físico, psicológico, sociocultural y cognitivo, que demandan de los jóvenes el desarrollo de estrategias de afrontamiento que les permitan establecer un sentido de identidad, autonomía y éxito personal social (3).

Las personas que sufren depresión durante la adolescencia y que tienden a desarrollar problemas psiquiátricos durante su adultez temprana se han caracterizado como jóvenes que presentan episodios depresivos más severos, es decir, de mayor duración, con múltiples episodios, mayor número de síntomas e historia de intentos de suicidio (4) Además, se ha encontrado que los casos que no se diagnosticaron ni trataron síntomas depresivos en la adolescencia tienen una mayor probabilidad de presentar en la adultez temprana un elevado índice de depresión, funcionamiento psicológico y social adverso, y abuso de substancias. La violencia intrafamiliar, el sexo femenino, entre otros, son factores que frecuentemente se relacionan con ella como indican los estudios de Gómez Restrepo (5). En el estudio de Gómez Restrepo (5) con 181 alumnos de 5 Centros Educativos del sur de Madrid, se encontró mayor prevalencia de sintomatología depresiva en niñas que en niños (6).

En Paraguay, un estudio reveló que el $36,5 \%$ de un grupo de 323 adolescentes embarazadas tenía síndrome depresivo, 32,7\% de las cuales había recibido castigos físicos de sus padres durante la adolescencia y la depresión se vinculó a los bajos ingresos. (7). Otro estudio con 304 mujeres puérperas de un hospital público evidenció una asociación entre la violencia física, psicológica y el abuso sexual con episodios depresivos de tipo leve $(37,8 \%)$, moderado $(19 \%)$ y grave $(23,6 \%)$ entre otros $(8)$. Y en otra investigación con muestreo probabilístico en población de estudiantes, la depresión recayó mayoritariamente en varones más que en mujeres (9).

En Paraguay, aún se cuenta con datos limitados sobre factores asociados a la depresión, de ahí la importancia de este estudio. Es por ello que se propuso este estudio para identificar la presencia de sintomatología asociada al síndrome depresivo en adolescentes de 56 colegios públicos y privados de Alto Paraná.

\section{MATERIALES Y MÉTODO}

Se realizó un estudio transversal descriptivo en adolescentes de 56 colegios de las 4 ciudades más pobladas del departamento de Alto Paraná (Hernandarias, Presidente Franco, Minga guazú, Ciudad del Este). El muestreo fue probabilístico y trietápico. Se completó un cuestionario auto-administrado, estructurado y anónimo, previa autorización de los padres. Para conocer la sintomatología referida al síndrome depresivo se utilizaron 20 preguntas referidas a niveles de malestar psíquicos utilizadas en la encuesta nacional 
"Salud Mental y Hábitos tóxicos en Paraguay" (10). La presencia de 8 síntomas como minino fue indicador de la presencia de síndrome depresivo. Las variables estudiadas fueron sexo, edad, antecedente de violencia psicológica, antecedente de violencia física, intento de abuso sexual o abuso consumado. Para poder identificar a aquellos estudiantes que padecieron violencia física y psicológica, se utilizó la Escala de Tácticas de Conflicto de Gelles y Strauss (11), que mide desde empleo de la discusión racional y el consenso hasta el uso de la fuerza física más extrema. Se tomó la definición de abuso sexual utilizada por UNICEF en el 2000 (12) que lo define como "relaciones sexuales forzadas, impuestas mediante amenazas e intimidaciones o con la fuerza física, la coerción a prácticas sexuales indeseadas, o la constricción a tener relaciones sexuales con terceros"; Para determinar la violencia física las definiciones del Grupo de trabajo sobre maltrato infantil dependiente del Ministerio de Salud de Chile 2001 (13), expresada como: "violencia o maltrato físico "toda lesión física no accidental producto de castigo único o repetido de magnitud y características variables"; de tipo emocional entendido como "hostigamiento verbal, habitual de un niño a través de insultos, criticas, descréditos y ridiculización, así como la indiferencia o rechazo implícito o explícito". A esto se le agregaron las variables socio económicas el grupo etário, sexo.

El instrumento que contenía las variables mencionadas fue un cuestionario autoadministrado, estructurado y anónimo. Previa autorización del Ministerio de Educación y Cultura merced a su máxima autoridad, autorización escrita recibida de parte de los padres de los estudiantes, se invitó a los jóvenes a participar en forma voluntaria, proporcionando información suficiente y clara sobre la utilización confidencial de los datos y fines de la investigación. La información fue cargada en una base de datos en Excel y analizada con SPSS 15.0 para Windows.

\section{RESULTADOS}

El 35,8\% $(n=237)$ de los 661 estudiantes presentaba sintomatología depresiva en base a la presencia de 8 síntomas. Tabla 1.

Tabla 1. Frecuencia de síndrome depresivo. $n=661$

\begin{tabular}{lll}
\hline Síntomas & Con síndrome depresivo & \multicolumn{1}{c}{ Sin síndrome depresivo } \\
\hline Presencia de 8 o más síntomas & $237(35,9)$ & $424(64,1)$ \\
Presencia de 11 o más síntomas & $104(15,7)$ & $557(84,3)$ \\
\hline
\end{tabular}

En el análisis bivariado, los factores asociados a la depresión definida tanto por ocho síntomas como por once síntomas fueron sexo femenino ( $p<0,001 ; R R: 2)$, antecedente de violencia física $(p<0,001 ; R R: 1,86)$, antecedente de violencia psicológica $(p<0,001$; RR: 2,7$)$, intento de abuso sexual o abuso consumado ( $p<0,001 ; R R: 1,6)$. Tabla 2. 
Tabla 2. Factores asociados al síndrome depresivo con base en la presencia de 8 síntomas en adolescentes de colegios públicos y privados de Alto Paraná. Análisis bivariado

\begin{tabular}{llll}
\hline Factores asociados & Con depresión & RR & Valor p \\
\hline Sexo & $163 / 343(47,5)$ & 2 & $<0,001$ \\
$\quad$ Femenino & $74 / 244(23,3)$ & & \\
Masculino & & & \\
Violencia física & $178 / 419(42,5)$ & 1,86 & $<0,001$ \\
$\quad$ Presencia & $47 / 205(22,9)$ & & \\
$\quad$ Ausencia & & & \\
Violencia psicológica & $196 / 449(43,6)$ & 2,7 & $<0,001$ \\
$\quad$ Presencia & $28 / 174(16,1)$ & & \\
$\quad$ Ausencia & & & \\
Intento de abuso o abuso consumado & $82 / 160(51,3)$ & 1,6 & $<0,001$ \\
Presencia & $150 / 477(31,4)$ & & \\
Ausencia & & & \\
\hline
\end{tabular}

En el análisis multivariado los factores asociados fueron antecedente de violencia psicológica, el sexo femenino, intento de abuso sexual o abuso (Tabla 3 ).

Tabla 3. Factores asociados a la depresión. Análisis multivariado

\begin{tabular}{lc}
\hline Factores & $\begin{array}{c}\text { Depresión } \\
\text { OR síntomas/11 síntomas } \\
\text { OR }\end{array}$ \\
\hline Antecedentes de violencia psicológica & $4 / 4$ \\
Sexo femenino & $3,5 / 4$ \\
Intento de abuso o abuso consumado & $2 / 2,4$ \\
\hline
\end{tabular}

\section{DISCUSIÓN}

Los factores asociados a la depresión de este grupo de estudiantes, después del ajuste estadístico fueron la violencia psicológica, el sexo femenino y el abuso sexual de los cuales habían sido víctimas en el entorno familiar principalmente. Blazer et al. 1994 (14), citados por Cassano \& Fava (15) hallaron que en Estados Unidos la prevalencia del trastorno depresivo mayor a lo largo de la vida correspondía a $21,3 \%$ en mujeres y $12,7 \%$ en hombres, casi doblando el porcentaje. La evidencia de violencia asociada al género femenino en el presente estudio, vuelve a poner en relieve, como refieren investigaciones de violencia de género, que la abrumadora carga mundial de violencia aún continúa siendo mayoritariamente llevada por mujeres, en su mayoría jóvenes, con graves consecuencias para la salud mental. Esta carga de violencia experimentada aumenta sus probabilidades de desarrollar otro tipo de trastornos, como los de estrés post traumático, trastornos afectivos, trastornos por somatización a mediano y a largo plazo (16-21) lo que profundiza la desigualdad de oportunidades a futuro (22).

Si bien el presente estudio no utilizó categorías de severidad en la depresión encontrada (como leve, moderada o grave), los hallazgos coinciden con los estudios de Kaplan et al. de 1998 (23) que asociaron a los trastornos depresivos haber padecido abuso físico, percepción de sobreprotección y contar con sólo un padre biológico en el hogar. Este estudio también encontró una asociación entre depresión y maltrato físico y los jóvenes 
abusados físicamente tenían una probabilidad siete veces mayor de desarrollar un trastorno depresivo mayor, que aquellos jóvenes que no fueron maltratados. El estudio de McHolm et al. del 2003 (24), concluyó con el hallazgo de una asociación entre la exposición a uno o más tipos de maltrato en la infancia, (físico, sexual o negligencia) y depresión. McHolm et al. en el 2003 (24) realizaron un estudio con 347 mujeres de 15 a 64 años, $40.3 \%$ de las cuales presentaron trastorno depresivo mayor. Estas mujeres deprimidas que experimentaron abuso físico en la niñez mostraron una probabilidad casi tres veces mayor de tener ideación suicida lo largo de su vida.

Estudios de Aalto-Setala et al. (4), en el 2002 refuerzan lo evidenciado en este estudio al reportar que síntomas depresivos reportados en la adolescencia aumentaron el riesgo de trastorno psiquiátrico y consumo de alcohol en la adultez temprana, con el riesgo tres veces mayor de presentar trastorno depresivo (trastorno depresivo mayor o distimia), o problemas psicosociales. También el estudio realizado en Paraguay en 304 puérperas con antecedentes de violencia, coinciden con los hallazgos de una asociación significativa entre padecimiento de violencia física, psicológica y abuso sexual con episodios depresivos (8) aunque éste se realizó con muestreo consecutivo. Al igual que los estudios de Vitriolo en una unidad psiquiátrica en el 2006 con el hallazgo de estrés post traumático de aparición tardía provocado por el abuso sexual en la infancia (16).

La depresión se ha convertido en un problema de salud pública cada vez mayor, que requiere de políticas públicas que aborden la prevención de la violencia intrafamiliar y la violencia de género como factores propiciadores de la misma durante el proceso adolescente en Paraguay, tal como muestran las evidencias científicas respecto a su incidencia.

Aunque podemos afirmar que han aumentado los datos científicos en la mayoría de las regiones sobre la prevalencia, las consecuencias y los factores de riesgo que se han asociado con la violencia, es necesaria mayor información. Por ejemplo, ahondar en estudios que aporten mayor información sobre la violencia en la esfera comunitaria y social, como las normas de género y la desigualdad de género y sus repercusiones a nivel social y comunitario. Importan principalmente, las repercusiones en la educación y salud de los niños y niñas. $(17,25)$. Estas recomendaciones han sido remarcadas principalmente para países como Paraguay, de medianos y bajos ingresos (25). La ejecución de programas escolares de prevención de la violencia en citas amorosas ha sido señalada por organismos internacionales así como la modificación de normas sociales y culturales relacionadas con el género (25) como por ejemplo, intervenciones educativas que apunten a descolocar afirmaciones y creencias que sostienen la violencia de género y la falta de autonomía en la mujer, basados principalmente en modificar las normas patriarcales y de predominio masculino vigentes en Paraguay $(26,27)$.

La violencia sexual que causa depresión en las mujeres es una expresión de poder y no de sexualidad, pues atenta contra la voluntad, la integridad y la dignidad de otra persona, es dable entender que la violencia por razones de género o la "violencia sexual contra las mujeres, los chicos y las chicas no es la expresión agresiva de la sexualidad sino la expresión sexual de la agresión, hostilidad y poder con una base en las condiciones del patriarcado" (28). Construir una cultura de paz representa una concepción centrada en la abolición de las relaciones de poder entre géneros, sin que ello signifique ausencia de conflicto, sólo será posible con la eliminación de la opresión y la injusticia. $(28,29)$

\section{REFERENCIAS BIBLIOGRAFICAS}

1. Pardo G, Sandoval A, Umbarila D. Adolescencia y depresión. Revista Colombiana de Psicología. 2004;13(1):13-28.

2. Gómez C, Rodríguez N. Factores de riesgo asociados al síndrome depresivo en la población colombiana. Revista Colombiana de Psiquiatría. 1997; 26(1):23-35.

3. Blum, R. (2000). Un modelo conceptual de salud del adolescente. En E. Dulanto (Ed.), El adolescente (pp. 656-672). Mexico: Mc Graw Hill.

4. Aalto-Setala $T$, Marttunen $M$, TuulioHenriksson A, Poikolainen K, Lonnqvist $\mathrm{J}$. Depressive symptoms in adolescence as predictors of early adulthood depressive disorders and maladjustment. The American Journal of Psychiatry. 2002;152:1235-7.

5. Gómez-Restrepo C, Bohórquez A, Pinto Masis D, Gil Laverde JFA, Rondón Sepúlveda $M$, Díaz-Granados N. Prevalencia de depresión y factores asociados con ella en la población colombiana. Rev Panam Salud Publica. 2004;16(6):378-86

6. Martínez Otero V. Sintomatología depresiva en niños: estudio de una muestra de escolares de la zona sur de Madrid Capital. Revista Electrónica de Psicología Iztacala 2007;10(3):73-88. 
7. Arrom C, Ruoti M, Samudio M, Orué E. Condiciones socioeconómicas de adolescentes embarazadas con síntomas de depresión, consumo de sustancias y víctimas de violencia intrafamiliar. Revista Paraguaya de Psiquiatría. 2015;3(1):13-9.

8. Arrom C, Samudio M, Arrom CM, Lampert N, Arrom MA, Orué E. Violencia intrafamiliar, de pareja íntima y abuso sexual en puerperio inmediato. Su relación con depresión. Revista Paraguaya de Psiquiatría. 2013;1(2):17-23.

9. Arrom C, Ruoti $M$, Samudio M, Orué E. Sintomatología depresiva y su relación con la edad y el sexo en adolescentes estudiantes de colegios secundarios de Alto Paraná. Revista Paraguaya de Psiquiatría. 2015;3(1):33-7.

10. Míguez H., Pecci M., Carrizosa A. Estudio sobre salud mental y hábitos tóxicos en el Paraguay. Proyecto Marandú del Comité Paraguay Kansas. Ministerio de Salud Pública y Bienestar Social. Asunción-Paraguay: Comité Paraguay Kansas. 1991.

11. Gelles R., Straus M. Intimate Violence. The definitive study of the causes and consequences of abuse in the american family. 1988. Published by the Simon \& Schuster Trade Division. Manufactured in the United States of America.

12. UNICEF [Internet]. Florencia-Italia: Innocenti Digest-Unicef; [citado 2 abril 2015]. Disponible en: http://www.unicefirc.org/publications/pdf/digest6s.pdf

13. UNICEF [Internet]. Asunción-Paraguay: Base Educativa y Comunitaria de Apoyo (BECA), UNICEF; [citado 11 de junio 2013]. Disponible en PDF: http://www.unicef.org/paraguay/spanish/py_ resources_Estudio_Maltrato.pdf

14. Blazer DG, Kessler RC, McGonnagle KA, Swartz MS. The prevalence and distribution of major depression in national community sample: The National Comorbidity Survey. Am J Psychiatry. 1994;151(7):979-86.

15. Cassano P, Fava M. Depression and public health: An overview. Journal of Psychosomatic Research. 2002;53:849-57.

16. Weil K, Florenzano R, Vitriol V, Cruz C, Carvajal C, Fullerton C, Muñiz C. Trauma infanto juvenil y psicopatología adulta: un estudio empírico. Rev Méd Chile 2004;132:1499-504.

17. Organización Mundial de la Salud-Oficina Regional para las Américas-Organización Panamericana de la Salud [Internet]. Washington D.C., EEUU: OPS-OMS; [citado 2 abril 2014]. Disponible en: http://apps.who.int/iris/bitstream/10665/988 16/1/WHO_RHR_12.36_spa.pdf?ua $=1$

18. Pereda N, Gallardo-Pujol D, Padilla R. Trastornos de personalidad en víctimas de abuso sexual infantil. = Personality disorders in child sexual abuse victims. Actas Españolas De Psiquiatría [serial on the Internet]. (2011, Mar), [cited August 12, 2015]; 39(2):131-9. Available from: PsycINFO.
19. Fresco MP, Arrom C, Samudio M, Arrom CM, Capurro M, Arrom MA, Arce A, Fresco $M$. Psicopatología en adultos con antecedentes de violencia intrafamiliar. Revista Paraguaya de Psiquiatría. 2015;3(1):8-12.

20. Cynthia L. Battle C, M. Tracie Shea, Dawn M. Johnson, Shirley Yen, Caron Zlotnick, Mary C. Zanarini, et al. Childhood Maltreatment Associated with Adult Personality Disorders: Findings from the Collaborative Longitudinal Personality Disorders Study. Journal of Personality Disorders. 2004,18(2):193-211.

21. Cortés D, Justicia F. Afrontamiento del abuso sexual infantil y ajuste psicológico a largo plazo. = Child sexual abuse coping and long term psychological adjustment. Psicothema [serial on the Internet]. (2008, Nov), [cited August 12, 2015]; 20(4):509-15. Available from: PsycINFO

22. Chavez Ayala R, Rivera-Rivera L, Llerenas A, Díaz-Cerón $\mathrm{E}$, Allen-Leigh $\mathrm{B}$, Lazcano Ponce E. Factores del abuso sexual en la niñez y la adolescencia en estudiantes de Morelos, México. Rev Saúde Pública 2009;43(3):50614.

23. Kaplan, S., Pelcovitz, D., Salzinger, S., Weiner, M., Mandel F., Lesser, M., \& Labruna, V. (1998). Adolescent physical abuse: Risk for adolescent psychiatric disorders. The American Journal of Psychiatry, 155(7):95460.

24. McHolm, A., MacMillan, H., \& Jamieson, E. The relationship between childhood physical abuse and suicidality among depressed women: Results from a community sample The American Journal of Psychiatry, 2003;160(5):933-8.

25. Organización Mundial de la Salud-Escuela de Higiene y Medicina Tropical de Londres. [Internet]. Washington D.C.: OPS-OMS; [citado 10 Mayo 2014]. Disponible en: http://apps.who.int/iris/bitstream/10665/448 10/1/9789275316351_spa.pdf

26. Arrom CM, Fresco MP, Arrom MA, Arrom C y cols. Afirmaciones y creencias sobre violencia de género y autonomía de la mujer en población consultante. Estudio comparativo. Revista Científica Estudios e Investigaciones, UNIBE. 2015;4(1):9-20.

27. Torales J. La violencia contra las mujeres es un problema de Estado: Programa de 14 puntos para la prevención, sanción y erradicación de la violencia contra las mujeres. Revista Paraguaya de Psiquiatría. 2015;3(1):70-3.

28. Barragan Medero F. Educación, adolescencia y violencia de género: Les amours finissent un jour. Universidad de La Laguna, Tenerife, España. 2006;6(1):31-53.

29. González Vera, M. Patriarcado, machismo e impunidad: causas de la cotidiana violencia de género En: Coordinadora de Derechos Humanos del Paraguay (Codehupy). Derechos humanos en Paraguay/ Yvypóra Derécho Paraguáipe. Codehupy. Asunción: Mercurio S.A.; 2012. pp. 447-463. 